\title{
A General Approach of Quasi-Exactly Solvable Schrödinger Equations with Three Known Eigenstates
}

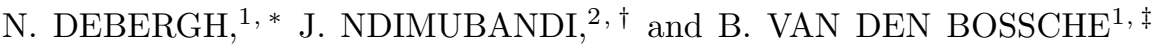 \\ ${ }^{1}$ Fundamental Theoretical Physics, Institute of Physics (B5), University of Liège, B-4000 LIEGE (Belgium) \\ ${ }^{2}$ University of Burundi, Department of Mathematics, P.O. Box 2700, BUJUMBURA (Burundi)
}

(Dated: August 2002)

We propose a general method for constructing quasi-exactly solvable potentials with three analytic eigenstates. These potentials can be real or complex functions but the spectrum is real. A comparison with other methods is also performed.

${ }^{*}$ Chercheur, Institut Interuniversitaire des Sciences Nucléaires, Bruxelles E-mail: Nathalie.Debergh@ulg.ac.be

${ }^{\dagger}$ Electronic address: indimubandi@yahoo.fr

${ }^{\ddagger}$ Electronic address: 


\section{INTRODUCTION}

Quasi-exactly solvable (Q.E.S.) Schrödinger equations are of great current interest. Indeed they have the characteristic property that analytic expressions for a finite number of energy levels and their corresponding eigenfunctions can be obtained. These Q.E.S. equations have been first investigated in [1] and since then they have been studied extensively (for a detailed review see f.i. [2]). Actually one can find two different points of view for analyzing these equations.

First, the number of analytic solutions is related [3] to the dimension of the irreducible representations of the Lie algebra $s l(2, R)$. More specifically the corresponding Schrödinger Hamiltonians can be written, up to a change of variables and a change of eigenfunctions, as linear and quadratic combinations of the $\operatorname{sl}(2, R)$ generators, these preserving the finite-dimensional space of the solutions.

Second, the number of analytic solutions is fixed at the start (essentially two [4] or three [5]). Except in the case of two solutions [ [ $]$ ], the corresponding Schrödinger Hamiltonians cannot, in general, be related to any Lie algebra.

Each of these two points of view has its own advantages and disadvantages. The Lie algebraic approach is elegant, straightforward and the associated potentials can be treated in a global way. However it concerns only a few interactions which have been listed in [3]. The second approach deals with a bigger and not yet known number of potentials but no general method to study them is available. Until now, the only method found in the literature to construct these Q.E.S. potentials is the one developed in [4] and [5]. It is based on a recursive construction of a finite number of supersymmetric partners [7]. It will be recalled in Section [V] with more details but let us already mention that the Kuliy-Tkachuk method is too constraining and thus misses some Q.E.S. interactions. Some of them are given in the following.

The purpose of this paper is then to propose an alternative to this method in order to approach the Q.E.S. equations with a known number (we will concentrate essentially on three) of analytic solutions in a general way. To do so we will make use of the general algorithm developed in 8 ].

The paper is organized as follows: In Section II, we explain the formalism of our method. It is illustrated through examples in Section III. In Section IV, we compare our method with the one of Kuliy and Tkachuk. Finally, we draw our conclusions in Section V.

\section{THE GENERAL APPROACH}

We concentrate on the one-dimensional and time-independent Schrödinger equation

$$
\left[-\frac{d^{2}}{d x^{2}}+V(x)\right] \psi_{N}(x)=E_{N} \psi_{N}(x) .
$$

The eigenfunctions $\psi_{N}(x)$ as explained in [8] are written as

$$
\psi_{N}(x)=g(x)[f(x)]^{\lambda_{N}} \sum_{m=0}^{N} c_{m}^{(N)}[h(x)]^{m}
$$

where the function $g(x)$ is a weight factor, $[f(x)]^{\lambda_{N}}$ is introduced due to eventual singularities in $V(x)$, and the quantities (real or complex) $c_{m}^{(N)}$ are expansion coefficients on the basis $h(x)$. Actually this basis plays the prominent role in our approach in the sense that each of the functions entering our formalism can be expanded in this basis according to

$$
\begin{aligned}
g^{\prime}(x) & =-g(x) \sum_{l=0}^{M} g_{l}^{1}[h(x)]^{l}, \\
f(x) & =\sum_{l=0}^{M} f_{l}^{0}[h(x)]^{l}, \\
f^{\prime}(x) & =\sum_{l=0}^{M} f_{l}^{1}[h(x)]^{l}, \\
h^{\prime}(x) & =\sum_{l=0}^{M} h_{l}^{1}[h(x)]^{l},
\end{aligned}
$$


the prime standing for the derivative with respect to $x$. The upper-right index for the coefficients $g_{l}^{1}, f_{l}^{0}, f_{l}^{1}, h_{l}^{1}$ indicates the derivative order of the corresponding expanded function. The upper summation index $M$ is in general an arbitrary positive integer, big enough to be the highest value of all upper summation indexes in Eqs. (3)-(6), the added expansion coefficients being vanishing when necessary. Finally the upper summation index $N$ is also a positive integer left arbitrary until we consider specific examples in the next Section.

In [8], we have limited ourselves to specific potentials of the form

$$
V(x)=\sum_{k=-V_{\min }}^{V_{\max }} V_{k}[f(x)]^{k} \sum_{l} v_{l, k}[h(x)]^{l}
$$

the coefficients $V_{k}$ and $v_{l, k}$ being fixed according to the interaction we want to deal with while $l$ runs in the space of integers. The positive integers $V_{\min }$ and $V_{\max }$ are also determined once the interaction is given. The expansion (7) has been proposed to include all known Q.E.S. potentials. However, in the present paper, we do not have to ask that the potential obeys a given expansion due to the fact that the number of eigenfunctions is fixed at the start. (We will nevertheless see in the next Section that the determined potential will be of the type of Eq. (8).)

More precisely, if we consider three eigenstates as stated in the Introduction, we have two possibilities: Either these eigenfunctions are of the form given in Eq. (2) with $N=L, L+1, L+2$, or they coincide with Eq. (2) with the same fixed $N=L$, but different values of $c_{m}^{(N)}$ in order to have linearly independent polynomials in the basis $h(x)$. The analysis of already known Q.E.S. potentials reveals that the Kuliy-Tkachuk approach is included in the first possibility while the Lie algebraic one can be found in the second possibility.

In both cases, we have the first eigenstate equal to

$$
\psi_{L}(x)=g(x)[f(x)]^{\lambda_{L}} \sum_{m=0}^{L} c_{m}^{(L)}[h(x)]^{m}
$$

and, for simplicity, associated to the energy

$$
E_{L}=0
$$

Such a requirement determines the potential $V(x)$ up to the presence of parameters which will be fixed asking for the existence of the other solutions. More precisely, by inserting Eqs. (8) and (9) in Eq. (1) and using Eqs. (3)-(6), the potential is

$$
\begin{aligned}
& V(x)=\left\{f_{p}^{0} f_{q}^{0} c_{m}^{(L)}[h(x)]^{p+q+m}\right\}^{-1}\left\{f_{k}^{0} f_{l}^{0} g_{m}^{1} g_{n}^{1} c_{s}^{(L)}[h(x)]^{k+l+m+n+s}\right. \\
& -m f_{k}^{0} f_{l}^{0} g_{m}^{1} h_{n}^{1} c_{s}^{(L)}[h(x)]^{k+l+m+n+s-1}+\lambda_{L} l f_{l}^{1} h_{k}^{1} f_{m}^{0} c_{s}^{(L)}[h(x)]^{k+l+m+s-1} \\
& +\left(\lambda_{L}^{2}-\lambda_{L}\right) f_{k}^{1} f_{l}^{1} c_{s}^{(L)}[h(x)]^{k+l+s}-2 \lambda_{L} g_{k}^{1} f_{l}^{0} f_{m}^{1} c_{s}^{(L)}[h(x)]^{k+l+m+s} \\
& +2 \lambda_{L} s f_{k}^{0} f_{l}^{1} h_{m}^{1} c_{s}^{(L)}[h(x)]^{k+l+m+s-1}-2 s f_{k}^{0} f_{l}^{0} g_{m}^{1} h_{n}^{1} c_{s}^{(L)}[h(x)]^{k+l+m+n+s-1} \\
& \left.+f_{k}^{0} f_{l}^{0} h_{m}^{1} h_{n}^{1} c_{s}^{(L)} s(s-1+m)[h(x)]^{k+l+m+n+s-2}\right\} .
\end{aligned}
$$

In this expression and the following ones, we have, for simplicity, omitted all the summation indexes but it is understood that there is a summation on each repeated index (except $L$ ).

Now the potential of Eq. (10) has to be constrained so that there exist other solutions to it. For the first possibility mentioned above, i.e., the one including in particular the Kuliy-Tkachuk potentials, we just have to require that the functions (2) with $N=L+1$ and $N=L+2$ are solutions of Eq. (1) with $V(x)$ fixed according to Eq. (10). This gives rise to (two) systems of algebraic equations (instead of differential ones). This is the main advantage, besides its generality, of our approach based on the development of all functions in the $h(x)$-basis. These systems are specified by vanishing the different coefficients of linearly independent terms in

$$
\begin{aligned}
& -f_{k}^{0} f_{l}^{0} h_{m}^{1} h_{n}^{1} c_{s}^{(L)} c_{t}^{(N)} t(t-1+m)[h(x)]^{k+l+m+n+s+t-2}+2 t f_{k}^{0} f_{l}^{0} g_{m}^{1} h_{n}^{1} c_{s}^{(L)} c_{t}^{(N)}[h(x)]^{k+l+m+n+s+t-1} \\
& -2 \lambda_{N} f_{k}^{0} f_{l}^{1} t h_{m}^{1} c_{s}^{(L)} c_{t}^{(N)}[h(x)]^{k+l+m+s+t-1}+\left(\lambda_{L}-\lambda_{N}\right)\left\{f_{k}^{0} f_{l}^{1} h_{m}^{1} l c_{s}^{(L)} c_{t}^{(N)}[h(x)]^{k+l+m+s+t-1}\right. \\
& \left.-2 f_{k}^{0} g_{l}^{1} f_{m}^{1} c_{s}^{(L)} c_{t}^{(N)}[h(x)]^{k+l+m+s+t}+\left(\lambda_{L}+\lambda_{N}-1\right) f_{k}^{1} f_{l}^{1} c_{s}^{(L)} c_{t}^{(N)}[h(x)]^{k+l+s+t}\right\} \\
& +2 \lambda_{L} f_{k}^{0} f_{l}^{1} h_{m}^{1} s c_{s}^{(L)} c_{t}^{(N)}[h(x)]^{k+l+m+s+t-1}-2 f_{k}^{0} f_{l}^{0} g_{m}^{1} s h_{n}^{1} c_{s}^{(L)} c_{t}^{(N)}[h(x)]^{k+l+m+n+s+t-1} \\
& +f_{k}^{0} f_{l}^{0} h_{m}^{1} h_{n}^{1} s(s-1+m) c_{s}^{(L)} c_{t}^{(N)}[h(x)]^{k+l+m+n+s+t-2}-E_{N} f_{k}^{0} f_{l}^{0} c_{s}^{(L)} c_{t}^{(N)}(h(x))^{k+l+s+t}=0
\end{aligned}
$$


where $N=L+1, L+2$. It is clear that if we want to consider the case of two only eigenstates, we have to solve only one system, the one corresponding to $N=L+1$. We can also extend this approach to the case of more than three solutions. The potential of Eq. (10) is then more and more constrained until it is impossible to fulfill the constraints.

In what concerns the second possibility, i.e., the one associated to the Lie algebraic approach, we have to ask for the function in Eq. (2) to be a solution of Eq. (11) with $N=L$, together with other coefficients than $c_{m}^{(L)}$, say $\tilde{c}_{m}^{(L)}$. The system of constraints is now:

$$
\begin{aligned}
& -f_{k}^{0} h_{m}^{1} h_{n}^{1} c_{s}^{(L)} \tilde{c}_{t}^{(L)}(t-s)(t-1+m+s)[h(x)]^{k+m+n+s+t-2}+2 f_{k}^{0} g_{m}^{1} h_{n}^{1} c_{s}^{(L)} \tilde{c}_{t}^{(L)}(t-s)[h(x)]^{k+m+n+s+t-1} \\
& -2 \lambda_{L} f_{k}^{1} h_{n}^{1} c_{s}^{(L)} \tilde{c}_{t}^{(L)}(t-s)[h(x)]^{k+n+s+t-1}=0 .
\end{aligned}
$$

The number of independent sets of coefficients $\tilde{c}_{t}^{(L)}$ satisfying Eq. (12) gives the number of eigenstates, besides $\psi_{L}(x)$ given in Eq. (8).

\section{EXAMPLES}

Let us now illustrate our approach through specific examples. The point is simply to choose the function $f(x)$ in an arbitrary way and then to adjust the important basis function $h(x)$ so that Eqs. (4) -(6) are satisfied. Our recent analysis of Q.E.S. equations in [8] has revealed that the choice

$$
f(x)=1+x^{2}
$$

covers many Q.E.S. interactions. This, as well as $f^{\prime}(x)=2 x$, clearly suggests the basis function $h(x)=x$. From Eqs. (4)-(6), we get the non vanishing coefficients

$$
f_{0}^{0}=1, f_{2}^{0}=1, f_{1}^{1}=2, h_{0}^{1}=1 .
$$

For the first possibility, corresponding to the constraints in Eq. (11), let us fix $L=1$ and choose, without loss of generality, $c_{1}^{(1)}=1$. The potential of Eq. (10) then reads

$$
\begin{aligned}
V(x)= & g_{m}^{1} g_{n}^{1} x^{m+n}-m g_{m}^{1} x^{m-1}+\frac{4 \lambda_{1}^{2}-2 \lambda_{1}}{1+x^{2}}-\frac{4 \lambda_{1}^{2}-4 \lambda_{1}}{\left(1+x^{2}\right)^{2}}-\frac{4 \lambda_{1}}{1+x^{2}} g_{m}^{1} x^{m+1} \\
& +\frac{4 \lambda_{1} x}{\left(1+x^{2}\right)\left(c_{0}^{(1)}+x\right)}-2 \frac{g_{m}^{1} x^{m}}{c_{0}^{(1)}+x} .
\end{aligned}
$$

A rapid look at the first constraint, corresponding to $N=2$ in Eq. (11), shows that the running index $m$ has in fact to be restricted to the values $m=0$ and $m=1$. This first constraint is then a system of eight (nonlinear) equations for the eight unknowns $g_{0}^{1}, g_{1}^{1}, c_{0}^{(1)}, c_{0}^{(2)}, c_{1}^{(2)}, \lambda_{1}, \lambda_{2}, E_{2}$ if, once again, we take advantage of the fact that the eigenstates are fixed up to a normalization constant, so that $c_{2}^{(2)}$ can be chosen equal to 1 . The second constraint, corresponding to $N=3$, is a system of nine (nonlinear) equations for the nine unknowns $g_{0}^{1}, g_{1}^{1}, c_{0}^{(1)}, c_{0}^{(3)}, c_{1}^{(3)}, c_{2}^{(3)}, \lambda_{1}, \lambda_{3}, E_{3}$ with, for the same reason, $c_{3}^{(3)}=1$. Thus there is a total of seventeen equations for thirteen unknowns. Nevertheless, this system is rather easy to handle and furnishes two sets of solutions. The first set is the one corresponding to the well-known harmonic oscillator potential while the second one is

$$
\begin{aligned}
& g_{0}^{1}=0, g_{1}^{1}=\sqrt{c}, \lambda_{1}=\lambda_{2}=\sqrt{c}-\frac{1}{2}, \lambda_{3}=\frac{3}{2}-\sqrt{c}, \\
& E_{2}=2 \sqrt{c}, E_{3}=-8 c+12 \sqrt{c}, c_{0}^{(1)}=0, c_{0}^{(2)}=-1, c_{1}^{(2)}=0, \\
& c_{0}^{(3)}=0, c_{1}^{(3)}=\frac{1}{\frac{4}{3} c-2}, c_{2}^{(3)}=0
\end{aligned}
$$

with $c$ being

$$
c=\left\{-1+\sqrt{5} \cos \left[\frac{1}{3} \arctan \left(\frac{\sqrt{109}}{4}\right)\right]\right\}^{2}
$$


i.e., $c=1.119639$. The associated potential is, from Eq. 110,

$$
V(x)=c x^{2}-4 c-\sqrt{c}+\frac{8 c-4 \sqrt{c}}{1+x^{2}}-\frac{4 c-8 \sqrt{c}+3}{\left(1+x^{2}\right)^{2}}
$$

and is, to our knowledge, a new Q.E.S. potential. It admits three analytic (and physical) solutions given by

$$
\begin{aligned}
& \psi_{1}(x)=x\left(1+x^{2}\right)^{\sqrt{c}-\frac{1}{2}} \exp \left(-\frac{\sqrt{c} x^{2}}{2}\right), E_{1}=0, \\
& \psi_{2}(x)=\left(1-x^{2}\right)\left(1+x^{2}\right)^{\sqrt{c}-\frac{1}{2}} \exp \left(-\frac{\sqrt{c} x^{2}}{2}\right), E_{2}=2 \sqrt{c} \\
& \psi_{3}(x)=x\left[1-\left(2-\frac{4}{3} c\right) x^{2}\right]\left(1+x^{2}\right)^{-\sqrt{c}+\frac{3}{2}} \exp \left(-\frac{\sqrt{c} x^{2}}{2}\right), E_{3}=-8 c+12 \sqrt{c} .
\end{aligned}
$$

We remark that the same analysis, but with $L=0$, would have led to the potential given in [5]. Thus, our method includes the one described in that reference. Moreover, as we will show in the next Section, it is simpler (see the function $U(x)$ of Eq. (39) which would be needed to find the potential Eq. (18) and the eigenfunctions Eqs. (19)-(21)).

For the second possibility, corresponding to the constraints in Eq. (12), let us fix $L=2$, which is the minimal value for having at most three eigenstates in this case, together with $c_{L}^{(L)}=1$ and $\tilde{c}_{L}^{(L)}=1$. We have not found a new potential with three eigenstates. Only known ones like the harmonic oscillator or its supersymmetric partner [5] emerge. We have however found a new potential with two eigenstates. It is given by

$$
V(x)=\frac{x^{2}}{36}-\frac{1}{6}+\frac{4+2 i x}{3(x+i)^{2}}
$$

and the two eigenstates and related eigenvalues are

$$
\begin{aligned}
& \psi_{2}(x)=\left(3+2 i x+x^{2}\right)\left(1+x^{2}\right)^{-1} \exp \left(-\frac{x^{2}}{12}\right), E_{2}=0, \\
& \tilde{\psi}_{2}(x)=\left(-1+2 i x+x^{2}\right) \exp \left(-\frac{x^{2}}{12}\right), \tilde{E}_{2}=\frac{2}{3} .
\end{aligned}
$$

Thus, we have here an example of a complex but PT-symmetric [9] Q.E.S. potential, with real eigenvalues. This potential is new compared to the ones already studied through other developments [10].

To end this Section, we mention that the potentials Eq. (18) and Eq. (22) could be written as in Eq. (7), implying that, given the potentials, we could have found the eigenfunctions using the algorithm we have described in [8].

\section{COMPARISON WITH THE KULIY-TKACHUK APPROACH}

For completeness, let us briefly recall what the method of Kuliy-Tkachuk is. It is based on a recursive construction of supersymmetric partners. Indeed, the initial potential $V(x)$ is required to be written as

$$
V(x)=W_{L}(x)^{2}-W_{L}^{\prime}(x) .
$$

If such a $W_{L}(x)$ does exist, then the ground state and its energy are known

$$
\psi_{L}(x)=\exp \left[-\int W_{L}(x) d x\right], E_{L}=0 .
$$

Provided one can find two other functions $W_{L+1}(x)$ and $W_{L+2}(x)$ such that the Ricatti equations

$$
W_{k}^{2}(x)+W_{k}^{\prime}(x)+E_{k}=W_{k+1}^{2}(x)-W_{k+1}^{\prime}(x)+E_{k+1}, k=L, L+1
$$

are satisfied, two other solutions (respectively associated with $E_{L+1}$ and $E_{L+2}$ ) are available. They are given by

$$
\begin{aligned}
& \psi_{L+1}(x)=\left[-\frac{d}{d x}+W_{L}(x)\right] \exp \left[-\int W_{L+1}(x) d x\right] \\
& \psi_{L+2}(x)=\left[-\frac{d}{d x}+W_{L}(x)\right]\left[-\frac{d}{d x}+W_{L+1}(x)\right] \exp \left[-\int W_{L+2}(x) d x\right] .
\end{aligned}
$$


The main point of the Kuliy-Tkachuk approach is to reduce Eqs. 27) to only one equation

$$
\begin{array}{r}
W_{+}^{(L)}(x) W_{+}^{(L+1)}(x)\left[W_{+}^{(L+1)}(x)-W_{+}^{(L)}(x)\right]-\frac{d}{d x}\left[W_{+}^{(L)}(x) W_{+}^{(L+1)}(x)\right] \\
+\left(E_{L+2}-E_{L+1}\right) W_{+}^{(L)}(x)+E_{L+1} W_{+}^{(L+1)}(x)=0
\end{array}
$$

of the two functions

$$
W_{+}^{(k)}(x) \equiv W_{k}(x)+W_{k+1}(x), k=L, L+1 .
$$

Finally, Eq. (30) can be considered as an algebraic one in terms of $W_{+}^{(L)}(x)$, for instance, if the function

$$
U(x) \equiv W_{+}^{(L)}(x) W_{+}^{(L+1)}(x)
$$

is fixed arbitrarily at the start. The whole approach thus relies on an adequate choice of this function $U(x)$. Note that the authors of Ref. [5] have restrained themselves to the context of the functions $U(x)$ such that

$$
\begin{aligned}
& U(x) \leq 0 \text { if } x \in\left[\min \left(x_{0}, \tilde{x}_{0}\right), \max \left(x_{0}, \tilde{x}_{0}\right)\right], \\
& U(x)>0 \text { otherwise }
\end{aligned}
$$

where $x_{0}$ and $\tilde{x}_{0}$ are the two zeroes of $U(x)$ such that

$$
U^{\prime}\left(\min \left(x_{0}, \tilde{x}_{0}\right)\right)<0, \quad U^{\prime}\left(\max \left(x_{0}, \tilde{x}_{0}\right)\right)>0 .
$$

In [5], these conditions were fixed according to the requirement that none of the functions $W_{l}(x), l=L, L+1, L+2$ possesses singularities.

Now we concentrate on the determination of this $U(x)$ related to the examples developed in the previous Section.

In what concerns the potential Eq. (18) and its eigenstates (19)-(21), a comparison with Eqs. (26) and (28)-(29) gives rise to the identifications

$$
\begin{aligned}
W_{L}(x) & =\sqrt{c} x-\frac{(2 \sqrt{c}-1) x}{\left(1+x^{2}\right)}-\frac{1}{x} \\
W_{L+1}(x) & =\sqrt{c} x-\frac{(2 \sqrt{c}+1) x}{\left(1+x^{2}\right)}+\frac{1}{x} \\
W_{L+2}(x) & =\sqrt{c} x+\frac{(2 \sqrt{c}+1) x}{\left(1+x^{2}\right)}-\frac{1}{x}-\frac{2 x\left[(14 c-3 \sqrt{c}-15)+(68 c+12 \sqrt{c}-90) x^{2}\right]}{(34 c+6 \sqrt{c}-45) x^{4}+(14 c-3 \sqrt{c}-15) x^{2}-3 \sqrt{c}} .
\end{aligned}
$$

It is already evident that such functions will lead to a Q.E.S. potential excluded in the Kuliy-Tkachuk approach due to the singularity $x=0$ in Eqs. (36)-(38). Indeed the corresponding $U(x)$ can be obtained through Eqs. (31)-(32) and is

$$
U(x)=2 \sqrt{c} x^{2}\left(x^{2}-1\right) \frac{(-192 c-39 \sqrt{c}+255) x^{2}+2(-17 c+3 \sqrt{c}+15)}{(34 c+6 \sqrt{c}-45) x^{4}+(14 c-3 \sqrt{c}-15) x^{2}-3 \sqrt{c}} .
$$

By opposition to the $U(x)$ permitted in [5], this one admits one second-order zero point $(x=0)$ and two ordinary zero points $(x= \pm 1)$. Moreover $U(x)$ has no pole and is such that (compare with Eqs. (33)-(35))

$$
\begin{aligned}
U(x) \leq 0 & \text { if } x \in[-1,1], \\
U(x)>0 & \text { if } x \in]-\infty,-1[\cup] 1,+\infty[, \\
U^{\prime}(0) & =0, U^{\prime}(-1)<0, \quad U^{\prime}(1)>0 .
\end{aligned}
$$

Let us also stress the heaviness of the $U(x)$ given in Eq. (39): The least one can say is that it is not natural to think at the start of such a function while the choice of our function $f(x)=1+x^{2}$ is on the other hand quite straightforward. This is the main advantage of our approach: using $L=0$ as a starting point in Eq. (8), we could have obtained the Kuliy-Tkachuk solution of Ref. [5] doing the same type of easy calculations that we have performed here. We would have of course obtained the same results as has been used in that reference, i.e.:

$$
V(x)=\frac{3}{4} x^{2}+\frac{1}{2}(6-7 \sqrt{3})-\frac{2(-3+\sqrt{3})}{1+x^{2}}+\frac{2(-3+2 \sqrt{3})}{\left(1+x^{2}\right)^{2}},
$$




$$
\begin{aligned}
& \psi_{0}(x)=\left(1+x^{2}\right)^{(3-\sqrt{3}) / 2} \exp \left(-\frac{\sqrt{3}}{4} x^{2}\right), E_{0}=0, \\
& \psi_{2}(x)=x\left(1+x^{2}\right)^{(\sqrt{3}-1) / 2} \exp \left(-\frac{\sqrt{3}}{4} x^{2}\right), E_{1}=6-3 \sqrt{3}, \\
& \psi_{3}(x)=\left(1-x^{2}\right)\left(1+x^{2}\right)^{(\sqrt{3}-1) / 2} \exp \left(-\frac{\sqrt{3}}{4} x^{2}\right), E_{2}=6-2 \sqrt{3}
\end{aligned}
$$

On the contrary, it is doubtful that the function $U(x)$ of Eq. (39) could have been witnessed from the start, while, in our approach, it just consists in starting with $L=1 \mathrm{in} \mathrm{Eq.} \mathrm{(8).} \mathrm{It} \mathrm{is} \mathrm{also} \mathrm{interesting} \mathrm{to} \mathrm{remark} \mathrm{that} \mathrm{the} \mathrm{potential} V(x)$ of [5], and our potential of Eq. (18) have the same type of $x$-dependence: $V(x)=a+b x^{2}+c /\left(1+x^{2}\right)+d /\left(1+x^{2}\right)^{2}$, and admit three solutions. This shape of the potential is very rich, see e.g [8]. In fact it is even possible (apart from the harmonic oscillator $c=d=0$ ) to find an exactly solvable model, using $a=0, b=1 / 4, c=4, d=-8$. One may wonder why it is not just possible to study the Schrödinger equation with a potential of this general shape. We have shown in Ref. [8] that the constraints are extremely difficult to handle, especially when the number of solutions is growing.

For the potential of Eq. (22), the function $U(x)$ simply reduces to $W_{+}^{(L)}(x)$ as defined in Eq. (31) due to the fact that this potential has two eigenvalues only. We respectively have

$$
\begin{aligned}
W_{L}(x) & =\frac{x}{6}+\frac{1}{x+i}-\frac{1}{x+3 i}, \\
W_{L+1}(x) & =\frac{x}{6}-\frac{1}{x+i}+\frac{1}{x+3 i}-\frac{1}{x+4 i}
\end{aligned}
$$

evidently leading to

$$
U(x) \equiv W_{+}^{(L)}(x)=\frac{x}{3}-\frac{1}{x+4 i} .
$$

So once again singularities (in the complex plane) arise and the resulting $U(x)$ could not be found following the method of Ref. [5].

\section{Remarks concerning the choice of $U(x)$}

We have already seen that the choice of $U(x)$ is a non trivial task. We want to show here that the choice is also sensitive to small changes of parameters, and that care must be done to extract meaningful solutions, i.e., even if $U(x)$ allows to generate a well-behaved potential $V(x)$, the eigenfunctions obtained using the formalism are not physically suitable. We show this starting with the simple choice $U(x)=c x^{2}$, with $c$ a constant to be determined. To avoid a $\mathcal{O}\left(1 / x^{2}\right)$ singularity in the potential, one is forced to adjust the constant $c$ to $c=E_{1}\left(E_{2}-E_{1}\right)$, with $E_{0}=0$. Choosing $E_{1}=1, E_{2}=2$, so as to have an equally spaced spectrum, two potentials emerge: either $V(x)=\left(x^{2}+10\right) / 4$, or $\tilde{V}(x)=\left(x^{2}-2\right) / 4$, which are both harmonic oscillator potentials. Because there is only a translation between the two, one may believe that both are admissible. This is however not the case using the general method of Ref. [5]. Using the different functions $W_{L}, L=0,1,2$ which enter Eqs. (26)-(32), the case which would lead to $V(x)$ implies unbounded solutions Eq. (26), Eq. (28) and Eq. (29) at infinity. Only the case which lead to the potential $\tilde{V}(x)$ has $W_{L}$-functions which ensure that the eigenfunctions are physical. Moreover, changing minimally the problem leads often to the impossibility to solve it. For example, taking $U(x)=3 x^{2}$, corresponding with non equally spaced energy levels $E_{0}=0, E_{1}=3, E_{2}=4$, leads to well-defined potentials, because $W_{0}(x)$ behaves adequately. It is however impossible to find physically acceptable wave-functions due to singularities and bad behavior at infinity of $W_{1}(x)$ and $W_{2}(x)$.

Another remark concerns the class of functions given in Ref. [8], which is too restrictive. For example, the well-known [3] sextic oscillator

$$
V_{0}(x)=x^{6}-11 x^{2}+8
$$

admitting the three solutions

$$
\psi_{0}(x)=\left(1+4 x^{2}+2 x^{4}\right) \exp \left(-\frac{1}{4} x^{4}\right), E_{0}=0
$$




$$
\begin{aligned}
& \psi_{1}(x)=\left(2 x^{4}-3\right) \exp \left(-\frac{1}{4} x^{4}\right), E_{1}=8 \\
& \psi_{2}(x)=\left(1-4 x^{2}+2 x^{4}\right) \exp \left(-\frac{1}{4} x^{4}\right), E_{2}=16
\end{aligned}
$$

corresponds to

$$
U(x)=\frac{\left(2 x^{4}-3\right)\left(2 x^{4}-1\right)}{x^{2}} .
$$

This $U(x)$ does not enter the Kuliy and Tkachuk formalism.

We have also tried different other choices for $U(x)$, which do not belong to the class of Kuliy and Tkachuk. We have investigated the two simplest deviations of the quadratic choice: $U(x)=c\left(x^{2}-a^{2}\right)$ and $U(x)=c x^{2}\left(x^{2}-a^{2}\right)$, with both choices $a^{2}>0$ or $a^{2}<0$. The result is that it is possible to find solutions for the first case. These solutions are however not physical (they diverge at infinity). In the second case, it is possible to find physically acceptable solutions. However, it is not possible to express them analytically because the functions $W_{L}, W_{L+1}$ and $W_{L+2}$ which enter Eq. (26) and Eqs. (28)-29) are not analytically integrable.

\section{CONCLUSIONS}

In this paper, we have presented a powerful alternative to the method of Kuliy and Tkachuk of Ref. [5]. We have shown that the function $U(x)$ is, even in a case which differs minimally from the one treated in that reference, complicated and heavy to handle: simple $U(x)$ do not lead to Q.E.S. potential; other $U(x)$ may give a good potential, but not physically relevant solutions. In contradistinction, our method seems straightforward to apply and, by construction, leads to physical solutions.

[1] V. Singh, S.N. Biswas and K. Datta, Phys. Rev. 18 (1978) 1901.

[2] A.G. Ushveridze, Quasi-Exactly Solvable Models in Quantum Mechanics, IOP Publishing Ltd (Bristol, 1994); M. A. Shifman, ITEP Lectures on Particle Physics and Field Theory, vol. 62, World Scientific Publishing Co. Pte. Ltd (Singapore, 1999).

[3] A.V. Turbiner and A.G. Ushveridze, Phys. Lett. A126 (1987) 181;

A.V. Turbiner, Comm. Math. Phys. 118 (1988) 467.

[4] V.M. Tkatchuk, Phys. Lett. A245 (1998) 177;

S.N. Dolya and O.B. Zaslavskii, J. Phys. A34 (2001) 1981.

[5] T.V. Kuliy and V.M. Tkachuk, J. Phys. A32 (1999) 2157.

[6] Y. Brihaye, N. Debergh and J. Ndimubandi, Mod. Phys. Lett. A16 (2001) 1243.

[7] C.V. Sukumar, J. Phys. A19 (1986) 2297.

[8] N. Debergh, J. Ndimubandi and B. Van den Bossche, Annals of Physics 298 (2002) 361.

[9] C.M. Bender and S. Boettcher, Phys. Rev. Let. 80 (1998) 5243.

[10] B. Bagchi and C. Quesne, Mod. Phys. Lett. A17 (2002) 463. 